Scientific Paper

\title{
Verification of calculations carried out with the Eclipse treatment planning system
}

\author{
Habib AHMAD ${ }^{1}$, Misbah AHMAD ${ }^{1, a}$, Shahid ALI ${ }^{2}$, M. Rauf KHATTAK ${ }^{1}$, Wajeeha SHAHEEN ${ }^{1}$, Jawad A. GILANI ${ }^{3}$, \\ Khalil AHMAD ${ }^{4}$ \\ ${ }^{I}$ Institute of Radiotherapy and Nuclear Medicine (IRNUM), Peshawar, Pakistan \\ ${ }^{2}$ Department of Physics, Peshawar University, Peshawar, Pakistan \\ ${ }^{3}$ Institute of Nuclear Medicine Oncology and Radiotherapy (INOR,) Abbottabad, Pakistan \\ ${ }^{4}$ Pakistan Institute of Nuclear Science and Technology (PINSTECH), Islamabad Pakistan
}

${ }^{a}$ E-mail address: misbahirnum@gmail.com

(received 20 August 2017; revised 26 January, 14 March, and 21 May 2018; accepted 26 June 2018)

\begin{abstract}
The goal of radiotherapy is to deliver prescribed dose to the target volume and simultaneously minimize the dose to the healthy organs. The purpose of this work was to verify the accuracy of calculations carried out with a treatment planning system (TPS). Measurements carried out with thermoluminescence detectors (TLDs) were compared with doses calculated with TPS. Doses were measured and calculated both in the open beam's region and under individual blocks. Measurements were performed in the Randophantom. The work was carried out for photon beams generated in the Varian CLINAC 2100C accelerator. The maximum / minimum percentage differences between measured and calculated doses were $4.9 / 0.6 \%, 2.6 / 0 \%$, and $3.5 \% / 0.5 \%$ in open, shielded and partially shielded points, respectively. Differences between the measured and calculated doses were within acceptable limits.
\end{abstract}

Key words: treatment planning system; radiation dosimetry; thermoluminescence; shielding blocks.

\section{Introduction}

The ultimate goal of radiotherapy is to deliver a prescribed dose to the target volume with minimum dose to the healthy tissues. There is a high demand for accuracy in radiotherapy. Even small deviations of delivered dose from prescribed one may have significant influence on the probability of cure and probability of complications. The dose needed for the local control of the disease and the dose tolerated by healthy tissue provides a narrow therapeutic window. Studies had shown that variation of $5 \%$ in dose resulted in $10-20 \%$ change in the tumor control and in the normal tissue complication probabilities were from $20-30 \%[1,2]$. It is expected that the uncertainty of dose delivery should be less than 5\% (two standard deviations) [3]. ICRU Reports 50 and 62 recommended that the dose uniformity in the target organ must be within uncertainties range $-5 \%$ to $+7 \%$ [4]. Treatment planning is a multistep process, whose complexity depends on many factors. Therefore, verification of accuracy of dose delivery is essential in radiotherapy daily practice and allows to minimize the likelihood of accidental exposure [5, 6]. Some studies have been done for solving specific problems associated with TPS performance and dose calculation [7-9]. For the purpose of testing, commissioning and QA of TPS, IAEA has published technical Report 430 [10] that provides a large number of tests and procedures. TPSs use different algorithms including Pencil Beam Convolution (PBC), Analytic Anisotropic Algorithm (AAA) and Monte Carlo Simulation for planning [11,12]. A comprehensive document on the treatment planning quality assurance was also developed by Task Group 53 of the American Association of Physicists in Medicine. In this report it postulated to keep the overall accuracy of the dose delivery within $\pm 5 \%[13,14]$. In the process of treatment planning the dose distribution is optimize to deliver the prescribe dose to target volume, the advisable dose distributions with a given treatment strategy, to deliver maximum dose to the target and very low dose to the organs at risk (OAR) [15-17]. For saving the OARs the shape of the fields are conformed to the target volume with multileaf collimators or customized Cerrobend blocks [16,17]. Thermoluminescence dosimetry has been in use for many decades and has valuable applications in various fields, such as personal, environmental, medical, archeological and geological dating [18-20]. Dosimeters like thermoluminescent, photoluminescent, optically stimulated luminescent, metal oxide semiconductor field effect transistor, electronic portal imaging device, radio-chromic and photographic films are in use for the last few decades for radiation dosimetry [19-22]. Most often the thermoluminescent TLD-100 (LiF: Mg, Ti) is used. It has an effective atomic number of 8.2 nearly equal to that of the soft tissue (7.4); therefore, TLD-100 interacts with 
radiation in the same way as a tissue does. Due to the small size of the detectors, they can be placed easily at the required position inside the phantom [23, 24].

In the study performed by Terohid et al. [25], the absorbed dose to the thyroid during chest wall radiotherapy was estimated to be $8 \%$ of the incident dose, compared to the previously performed study by M. Butanec et al. [26]. Tahirkhani et al. [27] evaluated the physical characteristics of Cerrobend blocks in radiotherapy and showed that the Cerrobend blocks are as almost efficient as the Multi-Leaf Collimators (MLCs) for some cases to protect OARs.

In the present study doses measured with TLD detectors and calculated with a treatment planning system were compared. Doses were measured in the anthropomorphic Randophantom. Doses were calculated in the Eclipse treatment planning system (Varian).

\section{Materials and Methods}

\section{Randophantom}

For clinical test cases, the commercially available Randophantom was used. The Randophantom is made of a completely human equivalent material having bone, tissue, lungs and air gaps. It is composed of 35 slices with slice thickness of $2.5 \mathrm{~cm}$. Every slice contains a grid of holes in which TLDs can be slipped in for measuring the radiation doses at various locations.

\section{Dosimetry system}

Absorbed dose measurements were carried out with thermoluminescent dosimeters TLD-100 chips manufactured by Harshaw chemical Co, Solon, USA. Annealing of all the detectors were carried out at $400^{\circ} \mathrm{C}$ for one hour, followed by 2 hours stay at $100^{\circ} \mathrm{C}$ in an electric furnace. Afterwards, TLDs were kept at room temperature. The time between exposure and read was kept constant (48 hours) to minimize the fading effect.

\section{Linear Accelerator}

A high energy linear accelerator Varian CLINAC 2100C installed at Institute of Nuclear Medicine Oncology and Radiotherapy (INOR), Abbottabad, Pakistan, was used for dose delivery with photon energies of $6 \mathrm{MV}$ and $15 \mathrm{MV}$ photons.

\section{Calibration of the detectors}

The annealed TLDs were exposed to a known dose of 500 mGy from a 60Co source repeatedly in a Secondary Standard Dosimetry Laboratory, Institute of Nuclear Science and Technology (PINSTECH), Islamabad. The detectors were read out using Thermo Scientific Harshaw 4500 semiautomatic TLD reader installed at Radiation Dosimetry Group, PINSTECH. TLDs having nearly identical response were grouped together as group 1 (45 TLDs) group 2 (23 TLDs) and group 3 (13 TLDs). The linearity of these selected detectors were checkedby exposing these detectors to a range of doses from $200 \mathrm{mGy}$ to $2000 \mathrm{mGy}$ with a step of $200 \mathrm{mGy}$.

\section{Treatment sites}

For all the selected treatment sites i.e. Acute Lymphoblastic Leukemia (ALL), Naso-Pharyngeal Carcinoma (NPC) and Hodgkin Disease (HD), case-wise number of utilized TLDs are given in Table 1.

\section{Treatment plans}

A Randophantom was scanned with a Computed Tomography (CT) unit. The CT images were transferred to Eclipse TPS, installed at Institute of Radiotherapy and Nuclear Medicine, Pakistan through Digital Imaging and Communications in Medicine (DICOM). The head and neck cases were scanned with a slice thickness of $3 \mathrm{~mm}$; while, the pelvic, abdomen and chest cases were scanned with a slice thickness of $5 \mathrm{~mm}$. In all cases, Planning Target Volume (PTV) and OARs were marked according to ICRU 50 and 62 protocols. Customized shielding blocks were prepared using Styroformer (Huestis Medical Styrofoam cutter) machine installed at INOR. The dose was calculated according to IAEA, TRS, 398 with ionization chamber and electrometer (PTW: UNIDOS E Universal Dosimeter). All treatment plans were simulated with the conventional simulator. The detectors were placed at the selected slices in specified locations within the Randophantom.

\section{Dose Delivery and Measurement}

The plans were executed by irradiating the Randophantom using $6 \mathrm{MV}$ and $15 \mathrm{MV}$ photon energies. The Case 1 was irradiated with standard Prophylactic Cranial Irradiation (PCI) field with two parallel opposed fields $6 \mathrm{MV}$ photon beams of $22 \times 19.5 \mathrm{~cm}^{2}$. The organs at risk (OAR) were shielded with customized Cerrobend blocks. Similarly, the Case 2a was irradiated with two parallel opposed lateral beams of field size $19.4 \times 18 \mathrm{~cm}^{2}$ and one Anterior Lower Neck (Case 2b) of field size $25 \times 6 \mathrm{~cm}^{2}$. The Case $3 \mathrm{a}$ and $3 \mathrm{~b}$ were irradiated with $\mathrm{A} / \mathrm{P}$ Mantle field and A/P Inverted Y fields by using $15 \mathrm{MV}$ photon beams of field size of $34 \times 32 \mathrm{~cm}^{2}$, and $26 \times 20.5 \mathrm{~cm}^{2}$, respectively. The doses were normalized at the isocenter for each case. The detectors were placed in open, shielded and partially shielded positions within the irradiating volumes. The conventional dose delivery regime in radiotherapy was followed i.e. $200 \mathrm{cGy}$ per fraction, in all the selected sites.

Table 1. Case-wise number of TLDs utilized in the study of each case.

\begin{tabular}{ccc}
\hline \hline Case & Case Study & Number of TLDs \\
\hline Case 1 & ALL & 10 \\
Case 2a & NPC & 9 \\
Case 2b & AnteriorLowerNeck & 13 \\
Case 3a & Mantle Field HD & 27 \\
Case 3b & Inverted Y Field HD & 22 \\
Total & 5 & 81 \\
\hline \hline
\end{tabular}




\section{Results}

The three groups of TLDs used for determination of absorbed dose in the study possessed a linear response when exposed to a dose from $200 \mathrm{mGy}$ to $2000 \mathrm{mGy}$ with a step size of 200 mGy, as shown in Figure 1. It was found that the TLD responded linearly with a regression coefficient $\left(\mathrm{R}^{2}\right)$ of 0.966 , 0.990 and 0.991 , and exhibiting standard deviation of $\pm 8.1 \%$, $\pm 5.0 \%$ and $\pm 5.5 \%$ in the response of each batch of TLD, respectively. The calibration factors $(0.201,0.221$ and 0.244 $\mathrm{mGy} / \mathrm{nC}$ ) of each group have been obtained. The individual calibration factors were used for dose determination.

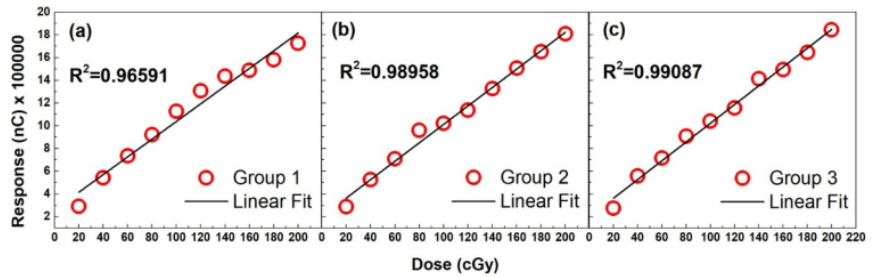

Figure 1. Linearity plots of TLD-100 for a) Group 1, b) Group 2, and c) Group 3.

\section{Case 1: Acute Lymphoblastic Leukemia}

In this case, the shielded organs (lens, sinuses, and orbit) doses, as recorded by the detectors are compared with the TPS point doses. Both were almost the same with negligible percentage difference, as shown in Table 2, and subsequently plotted in Figure 2. In the case of open target (unshielded), whole brain up to $\mathrm{C} 2$, the percentage difference between measured doses of

Table 2. Average response of the TLD and TPS point dose in cGy for Acute Lymphoblastic Leukemia (ALL).

\begin{tabular}{cccc}
\hline \hline $\begin{array}{c}\text { Position of } \\
\text { Dosimeters }\end{array}$ & $\begin{array}{c}\text { Average } \\
\text { Response of } \\
\text { Dosimeter }\end{array}$ & $\begin{array}{c}\text { Average Response } \\
\text { of Eclipse Point } \\
\text { Doses }\end{array}$ & $\begin{array}{c}\text { Percentage } \\
\text { Difference }\end{array}$ \\
\hline Open & $196.5(98.3 \%)$ & $191(95.5 \%)$ & 2.8 \\
Shielded & $20(10 \%)$ & $20(10 \%)$ & 0 \\
Partially Shielded & $166.5(83.3 \%)$ & $173(86.5 \%)$ & 3.3 \\
\hline \hline
\end{tabular}

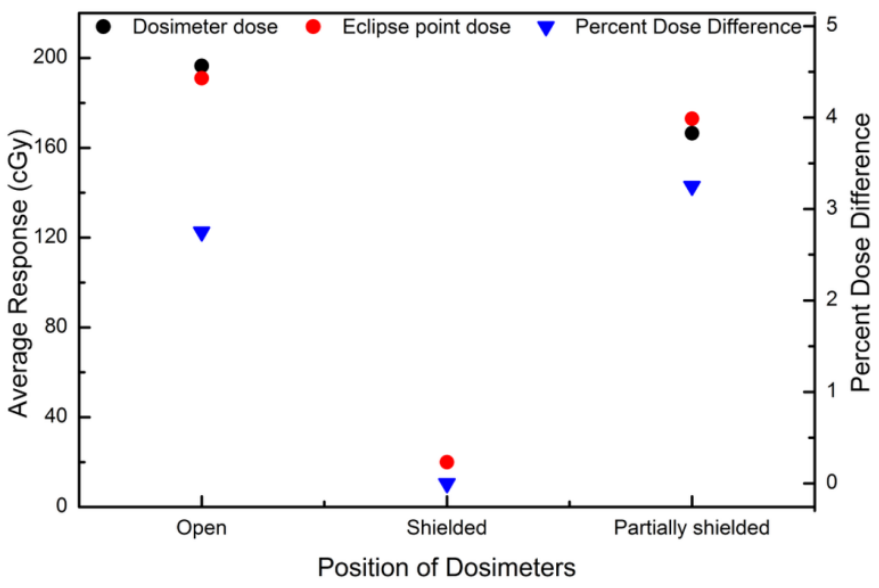

Figure 2. Average response of the dosimeter and TPS point dose for Acute Lymphoblastic Leukemia and their respective percentage difference. the detectors and the calculated dose by the TPS were $2.8 \%$, as shown in Table 2.

The percentage difference between TPS calculated and TLD measured doses were within the recommended limits, i.e. less than $5 \%$, which showed that the calculation agreed with the measurement. Moreover, in case of the partially shielded regions, the dose near the shielding blocks (area that is neither fully shielded nor fully open) as measured by the detectors and calculated by TPS were within the acceptable range of percentage difference i.e. $3.3 \%$.

\section{Case 2a: Naso-Pharyngeal Carcinoma}

The shielded organ dose measured by the detectors and Eclipse point doses of TPS were in good agreement with max. percentage difference of $1.5 \%$, confirming the excellent quality of fabricated blocks and accuracy of dose calculations. The target doses as measured by the detectors and calculated by TPS were also in good agreement with percentage difference of $3.0 \%$. Likewise, in the partially (near the edge of the shielding block) shielded sites the measured and calculated doses were also in a good agreement with a percentage difference of $0.5 \%$. All the recorded data have been reported in Table 3 and plotted in Figure 3.

\section{Case 2b: Anterior Lower Neck}

In order to treat the Naso-Pharyngeal Carcinoma, the whole neck nodes were to be irradiated. Therefore, direct Anterior Lower Neck field was added to the two parallel opposed fields. The 200 cGy per fraction dose was applied at Dmax.

Table 3. Average response of the dosimeter and TPS point doses in cGy for Naso-Pharyngeal Carcinoma.

\begin{tabular}{cccc}
\hline \hline $\begin{array}{c}\text { Position of } \\
\text { Dosimeters }\end{array}$ & $\begin{array}{c}\text { Average } \\
\text { Response of } \\
\text { Dosimeter }\end{array}$ & $\begin{array}{c}\text { Average Response } \\
\text { of Eclipse Point } \\
\text { Doses }\end{array}$ & $\begin{array}{c}\text { Percentage } \\
\text { Difference }\end{array}$ \\
\hline Open & $180.5(90.3 \%)$ & $174.5(87.3 \%)$ & 3.0 \\
Shielded & $12(6.0 \%)$ & $15(7.5 \%)$ & 1.5 \\
Partially Open & $41(20.5 \%)$ & $40(20.0 \%)$ & 0.5 \\
\hline \hline
\end{tabular}

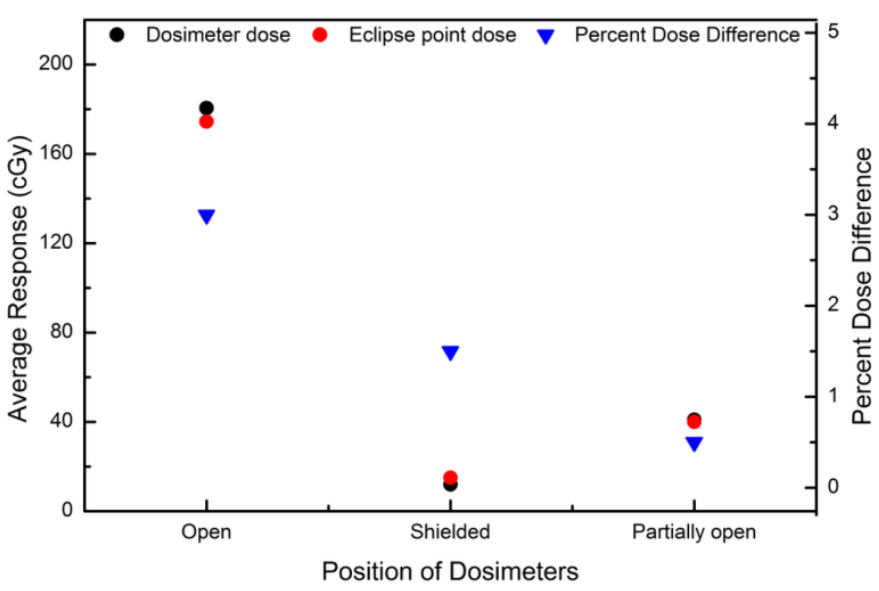

Figure 3. Average response of the dosimeter and TPS point dose for the Naso-Pharyngeal Carcinoma and their respective percentage difference. 
Table 4. Average response of the dosimeter and the TPS in cGy for Anterior Lower Neck.

\begin{tabular}{cccc}
\hline \hline $\begin{array}{c}\text { Position of } \\
\text { Dosimeters }\end{array}$ & $\begin{array}{c}\text { Average } \\
\text { Response of } \\
\text { Dosimeter }\end{array}$ & $\begin{array}{c}\text { Average Response } \\
\text { of Eclipse Point } \\
\text { Doses }\end{array}$ & $\begin{array}{c}\text { Percentage } \\
\text { Difference }\end{array}$ \\
\hline Open 3.5cm depth & $189.5(94.8 \%)$ & $179.8(89.9 \%)$ & 4.9 \\
Shielded & $17.5(8.8 \%)$ & $20(10 \%)$ & 1.3 \\
Partially Open & 0 & 0 & 0 \\
\hline \hline
\end{tabular}

Table 5. Average response of the dosimeter and the TPS point dose in cGy for Hodgkin's disease Mantle Field.

\begin{tabular}{cccc}
\hline \hline $\begin{array}{c}\text { Position of } \\
\text { Dosimeters }\end{array}$ & $\begin{array}{c}\text { Average } \\
\text { Response of } \\
\text { Dosimeter }\end{array}$ & $\begin{array}{c}\text { Average Response } \\
\text { of Eclipse Point } \\
\text { Doses }\end{array}$ & $\begin{array}{c}\text { Percentage } \\
\text { Difference }\end{array}$ \\
\hline Open & $188.7(94.3 \%)$ & $195.7(97.8 \%)$ & 3.5 \\
Shielded & $16.0(8 \%)$ & $20.5(10.3 \%)$ & 2.3 \\
Partially Open & 0 & 0 & 0 \\
\hline \hline
\end{tabular}

Table 6. Average response of the dosimeter and the TPS point dose in cGy for Hodgkin's disease for Inverted Y Field.

\begin{tabular}{cccc}
\hline \hline $\begin{array}{c}\text { Position of } \\
\text { Dosimeters }\end{array}$ & $\begin{array}{c}\text { Average } \\
\text { Response of } \\
\text { Dosimeter }\end{array}$ & $\begin{array}{c}\text { Average Response } \\
\text { of Eclipse Point } \\
\text { Doses }\end{array}$ & $\begin{array}{c}\text { Percentage } \\
\text { Difference }\end{array}$ \\
\hline Open & $193.2(96.6 \%)$ & $194.4(97.2 \%)$ & 0.6 \\
Shielded & $16.0(8.0 \%)$ & $21.2(10.6 \%)$ & 2.6 \\
Partially Open & $118.3(59.2 \%)$ & $111.3(55.7 \%)$ & 3.5 \\
\hline \hline
\end{tabular}

The detectors were placed at $3.5 \mathrm{~cm}$ depth in an open area that recorded dose of $189.5 \mathrm{cGy}$ by the TLDs and the Eclipse calculated point dose was $179.8 \mathrm{cGy}$. The percentage difference between these two systems was $4.9 \%$, which showed a good agreement. The dose measured by the detectors placed beneath the block and the Eclipse point doses beneath the blocks were also in good agreement with a percentage difference of $1.3 \%$, as presented in Table $\mathbf{4}$ and plotted in Figure 4.

\section{Case 3a: Hodgkin's disease Mantle Field}

To treat Hodgkin's diseases using Mantle Field customized blocks were used to shield both lungs. The doses recorded by the detectors and calculated by the Eclipse TPS under (beneath) the blocks showed a percentage difference of $2.3 \%$. Similarly, the measured and calculated dose in open (target) field was comparable with a percentage difference of $3.5 \%$, as shown in Table 5. The data has been plotted in Figure 5.

\section{Case 3b: Hodgkin's disease Inverted Y Field}

To treat the Hodgkin's disease in radiotherapy, another field i.e. invertedY Field is also applied. The customized blocks were used to protect theOARs. In this case, the OAR (bladder and kidneys) doses were determined by placing the detectors under the customized blocks in the corresponding slices. The difference between the measured and calculated dose was 2.6\%. Similarly, the target (open) doses and partially shielded doses measured with the detectors and calculated with TPS, were found in a good agreement with a percentage difference of $0.6 \%$ and $3.5 \%$, respectively, as shown in Table 6 and plotted in Figure 6.

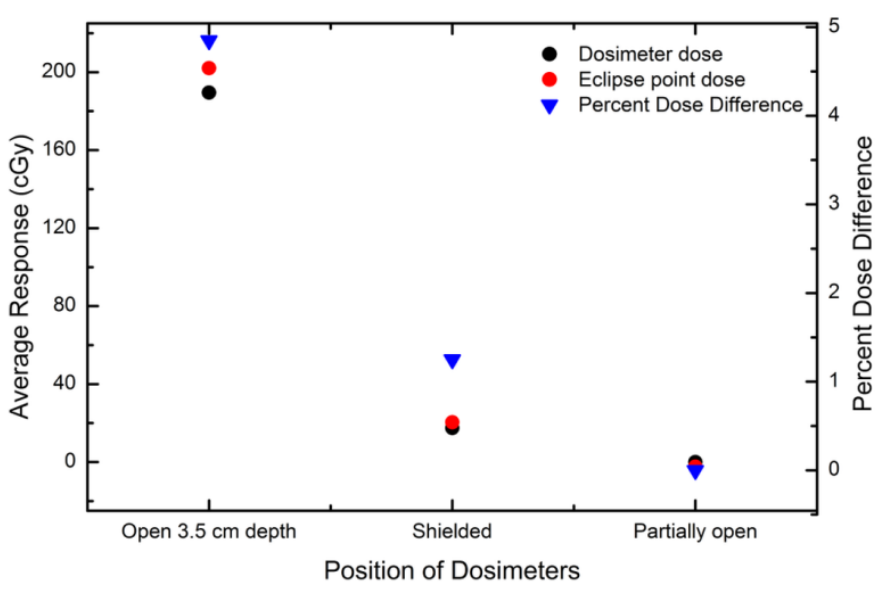

Figure 4. Average response of the dosimeter and the TPS for Anterior Lower Neck with their respective percentage differences.

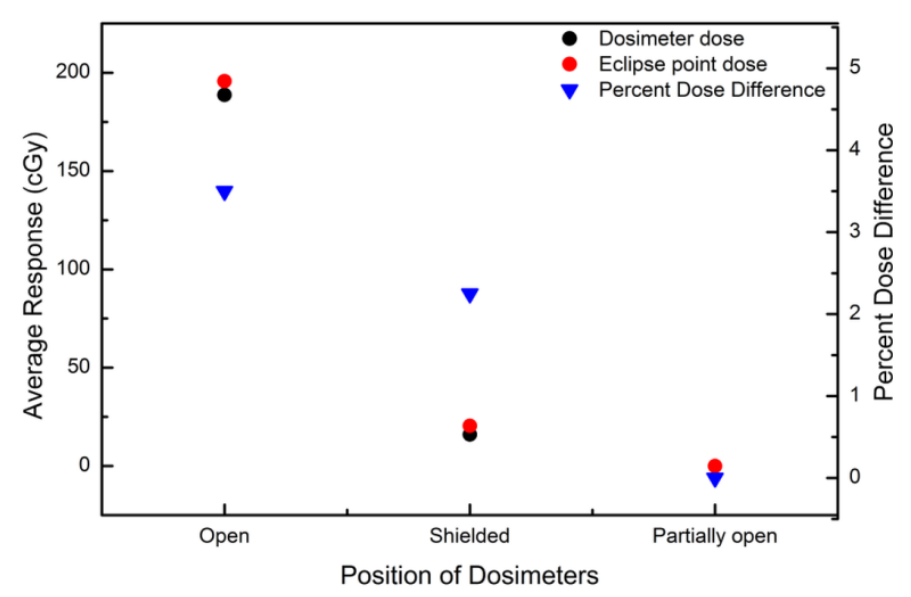

Figure 5. Average response of the dosimeter and the TPS for Hodgkin's disease Mantle Field with their respective percentage differences.

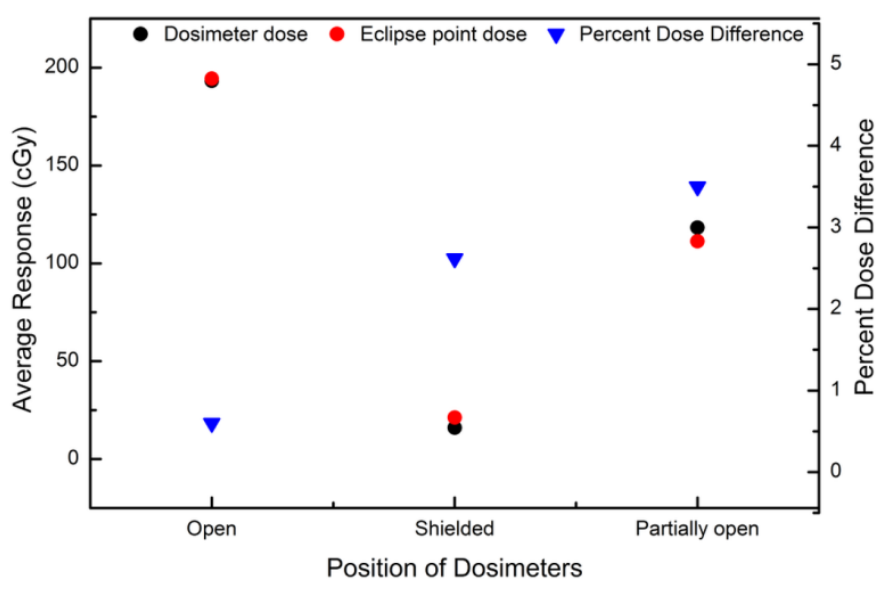

Figure 6. Average response of the dosimeter and the TPS for Hodgkin's disease Inverted $Y$ Field with their respective percentage differences. 


\section{Discussion}

Precise dose delivery in radiotherapy is crucial for the successful outcome of the treatment. Therefore radiation dose verification has become one of the top clinical priorities in the quality assurances for modern radiotherapy. In this work TLD100 were used for measurement of the doses and compared with the doses calculated by TPS. The results showed good agreement between the calculated and measured doses. The maximum differences between measured and calculated dose in open field were obtained for Case 1 (ALL) and Case 3b (Inverted Y). Comparison with TPS calculated doses revealed percentage differences of $2.8 \%$ and $0.6 \%$, respectively, as shown in Table 2 and Table 6. This showed a very good agreement between the TLD measured and the TPS calculated dose. The treatment of NPC showed a poorer agreement, with a percentage difference of $3 \%$ between measured and calculated dose in the target areas as shown in Table 3. This might be explained by using the customized blocks around the target.

To consider the next region (shielded region, maximum dose of $10 \%$ in case 1 was measured by the detector as well as calculated by the TPS, having the smallest percentage difference. The OARs in this case were the orbit and the lens of the eyes and received the dose far beyond its tolerance dose. In all the remaining cases, the percentage differences between measured and calculated doses were less than 3\%. The last region considered in the study was the partially shielded region dose. In this region, only three sites were selected to place the detectors in the vicinity of the blocks. The dose recorded in Case $2 \mathrm{a}$ by the detectors was $20.5 \%$ and calculated by TPS was $20 \%$, which have the least percentage difference of $0.5 \%$. In Case $3 \mathrm{~b}$, a moderate dose of $<60 \%$, was recorded, as shown in Table 6. The maximum recorded dose by TLDs and calculated by TPS was above $80 \%$ in Case1, indicating careful shielding of the OARs and careful irradiating the PTV to achieve maximum therapeutic benefits [28]. In the overall study, it was concluded that in most of the cases the dose measured by the detectors and calculated by the TPS under the blocks were in close agreement.

\section{Conclusions}

In this work, TLD-based procedure for the verification of various treatment plans used in radiotherapy was tested. The results of TLDs were compared with the doses calculated with Varian Eclipse TPS. The results were well within the acceptable limits.

\section{References}

[1] Brahme A. Dosimetric precision requirements in radiation therapy. Acta Radiol Oncol. 1984;23(5):379-391 .

[2] Thwaites D. Accuracy required and achievable in radiotherapy dosimetry: have modern technology and techniques changed our views? In: Journal of Physics: Conference Series, Vol. 444, IOP Publishing, 2013,p. 012006.

[3] IAEA. Absorbed dose determination in external beam radiotherapy: an international code of practice for dosimetry based on standards of absorbed dose to water. Vienna: International Atomic Energy Agency, Technical Reports Series TRS-398; 2000.

[4] ICRU. Prescribing, recording and reporting photon beam therapy (supplement to ICRU report 50), ICRU report 62; 1982.

[5] IAEA, Investigation of an accidental exposure of radiotherapy patients in Panama, International Atomic Energy Agency, 2001.

[6] IAEA. Lessons learned from accidental exposures in radiotherapy, IAEA Safety Reports Series 17; 59-61. 2000.

[7] Nisbet A, Beange I, Vollmar HS, et al. Dosimetric verification of a commercial collapsed cone algorithm in simulated clinical situations. Radiother Oncol. 2004;73(1):79-88 .

[8] Davidson SE, Ibbott GS, Prado KL, et al. Accuracy of two heterogeneity dose calculation algorithms for IMRT in treatment plans designed using an anthropomorphic thorax phantom. Med Phys. 2007;34(5):1850-1857.

[9] Cheng CW, Das IJ, Tang W, et al. Dosimetric comparison of treatment planning systems in irradiation of breast with tangential fields. Int J Radiat Oncol Biol Phys. 1997;38(4):835-842 .

[10] Van Dyk J. Quality assurance of radiation therapy planning systems: current status and remaining challenges. Int J Radiat Oncol Biol Phys. 2008;71(1):S23-S27.

[11] Alderson SW, Lanzl LH, Rollins M, et al. An instrumented phantom system for analog computation of treatment plans. Am J Roentgenol Radium Ther Nucl Med. 1962;87:185-195.

[12] Caprile PF, Venencia CD, Besa P. Comparison between measured and calculated dynamic wedge dose distributions using the anisotropic analytic algorithm and pencil-beam convolution. J Appl Clin Med Phys. 2007;8(1):47-54.

[13] Fogliata A, Vanetti E, Albers D, et al. On the dosimetric behaviour of photon dose calculation algorithms in the presence of simple geometric heterogeneities: comparison with Monte Carlo calculations. Phys Med Biol. 2007;52(5):1363-1385.

[14] Chavaudra J, Bridier A. Definition of volumes in external radiotherapy: ICRU reports 50 and 62. Cancer Radiothérapie. 2001;5(5): $472-478$.

[15] Essers M, Mijnheer B. In vivo dosimetry during external photon beam radiotherapy. Int J Radiat Oncol Biol Phys. 1999;43(2):245259. 
[16] Mijnheer BJ, Battermann JJ, Wambersie A. What degree of accuracy is required and can be achieved in photon and neutron therapy? Radiother Oncol. 1987;8(3):237-252.

[17] Ågren A, Brahme A, Turesson I. Optimization of uncomplicated control for head and neck tumors. Int J Radiat Oncol Biol Phys. 1990;19(4):1077-1085.

[18] McKeever SWS, Moscovitch M, Townsend PD. Thermoluminescence dosimetry materials: properties and uses. Nuclear Technology Publishing, Ashford, 1995.

[19] Ramani R, Russell S, O'Brien P. Clinical dosimetry using MOSFETs. Int J Radiat Oncol Biol Phys. 1997;37(4):959-964.

[20] Butson MJ, Rozenfeld A, Mathur JN, et al. A new radiotherapy surface dose detector: the MOSFET. Med Phys. 1996;23(5):655-658.

[21] Gladstone DJ, Chin LM. Real-time, in vivo measurement of radiation dose during radioimmunotherapy in mice using a miniature MOSFET dosimeter probe/ Radiat Res. 1995;141(3):330-335.

[22] Rosenblatt E, Zubizarreta E, Wondergem J, et al. The international atomic energy agency (IAEA): An active role in the global fight against cancer. Radiother Oncol. 2012;104(3):269-271.

[23] Izewska JE, Bera P, Vatnitsky S. IAEA/WHO TLD postal dose audit service and high precision measurements for radiotherapy level dosimetry. Radiat Prot Dosim. 2002;101(1-4):387-392.

[24] Ferreira IH, Dutreix A, Bridier A, et al. The ESTRO-QUALity assurance network (EQUAL) for European radiotherapy centers, in: Engineering in Medicine and Biology Society, 2000. Proceedings of the 22nd Annual International Conference of the IEEE,Vol. 4, IEEE, 2000, pp. 3112-3113.

[25] Terohid SA, Fayaz V. Estimating the absorbed dose to thyroid during chest wall radiotherapy. International Journal of Medical, Health, Biomedical,Bioengineering and Pharmaceutical Engineering. 2011;5(11):638-640.

[26] Budanec M, Bokulić T, Mrčela I, et al. Radiation treatment planning system verification. In: Proceedings International conference on quality assurance and new techniques in radiation medicine; Vienna (Austria); 13-15 Nov 2006.

[27] Farajollahi AR, Bouzarjomehri F, Kiani M. Comparison between clinically used irregular fields shielded by cerrobend and standard lead blocks. J Biomed Phys Eng. 2015;5(2):77-82.

[28] Khan FM, Gibbons JP. Khan's the physics of radiation therapy. Lippincott Williams \& Wilkins, 2014. 